\title{
Thermoelectric properties of finite two-dimensional quantum dot arrays with band-like electronic states
}

\author{
David M T Kuo \\ Department of Electrical Engineering and Department of Physics, \\ National Central University, Chungli, 320 Taiwan
}

(Dated: May 26, 2020)

\begin{abstract}
The thermal power $\left(P F=S^{2} G_{e}\right)$ depends on the Seebeck coefficient $(S)$ and electron conductance $\left(G_{e}\right)$. The enhancement of $G_{e}$ will unavoidably suppress $S$ because they are closely related. As a consequence, the optimization of $P F$ is extremely difficult. Here, we theoretically investigated the thermoelectric properties of two-dimensional quantum dot (QD) arrays with carriers injected from electrodes. The Lorenz number of $2 \mathrm{D}$ QD arrays in the resonant tunneling procedure satisfies the Wiedemann-Franz law, which confirms the formation of minibands. When the miniband center is far away from the Fermi level of the electrodes, the electron transport is in the thermionic-assisted tunneling procedure (TATP). In this regime, $G_{e}$ in band-like situation and $S$ in atom-like situation can happen simultaneously. We have demonstrated that the enhancement of $G_{e}$ with an increasing number of electronic states will not suppress $S$ in the TATP.
\end{abstract}

\section{INTRODUCTION}

The semiconductor quantum dots (QDs) resulting from the quantum confinement of heterostructures exhibit atom-like discrete electron energy levels. Highefficiency single-QD devices show the functionalities of low electrical and optical power outputs. These singleQD devices include single electron transistors [1-3], single photon sources [4, 6], single photon detectors [7] and single electron heat engines [8]. Some applications of QD devices require both high efficiency and significant output power. Therefore, one needs QD solids that can retain the size tunable properties of the QDs while exhibiting the band transport characteristic of bulk semiconductors. [9] Although much effort has been devoted to producing such QD solids, studies of the thermoelectric properties of such 2D QD arrays have been lacking. 10.11

Designing a thermoelectric material with a high figure of merit $(Z T)$ and optimized power output is under pursuit. 12-14] The dimensionless figure of merit $Z T=$ $S^{2} G_{e} T / \kappa$ depend on the Seeback coefficient $(S)$, electrical conductance $\left(G_{e}\right)$ and thermal conductance $(\kappa)$ of the material. Although 1D QD arrays have very high $Z T$ values, there exist many limitations in the implementations of thermoelectric devices. 9] 2D and 3D QD arrays are required for realistic applications. The $\kappa$ of a 2D QD array is smaller than that of bulk material. 15. This low dimensional system has the potential to realize high $Z T$ values. [16] Therefore, it is desirable to investigate the power factor $\left(P F=S^{2} G_{e}\right)$ of $2 \mathrm{D} \mathrm{QD}$ arrays, which directly affects the electrical power output. The enhancement of $G_{e}$ calls for a large number of electronic states (band-like). However a large $S$ value occurs in dilute electronic states (atom-like). Therefore, enhancing one of these physical quantities will unavoidably suppress the other. This study theoretically investigated the thermoelectric properties of a finite 2D QD array coupled to electrodes, as shown in Fig. 1. The electrons of the QD array are injected from the electrodes. 17] We demonstrated that $G_{e}$ in band-like situation and $S$ in atom- like situation can happen simultaneously when the miniband center of a 2D QD array remains a certain distance from the Fermi level of the electrodes. These results will improve the thermoelectric performance of $2 \mathrm{D}$ materials such as $S n S e$ and $M o S_{2} \cdot[18$.20]

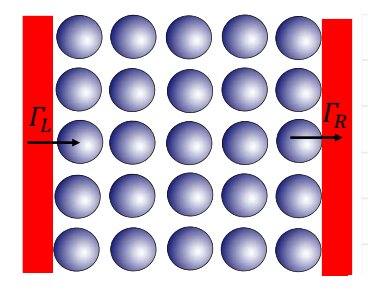

(a) 2-D QD ARRAY (b) Energy diagram

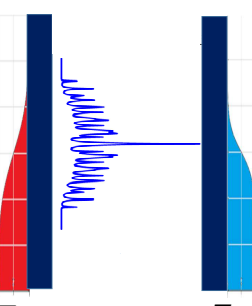

$T_{L}$
$T_{R}$
FIG. 1: (a) Schematic diagram of a two dimensional (2D) quantum dot (QD) array coupled to electrodes. $\Gamma_{L}\left(\Gamma_{R}\right)$ denotes the tunneling rate of the electrons between the left (right) electrode and the leftmost (rightmost) QDs. Energy diagram of a 2D QD array coupled to electrodes with different equilibrium temperatures $\left(T_{L}\right.$ and $\left.T_{R}\right)$.

\section{FORMALISM}

To model the thermoelectric properties of a 2D QD array connected to the electrodes, the Hamiltonian of the system shown in Fig. 1 is given by $H=H_{0}+H_{Q D}, 21$. where

$$
\begin{aligned}
H_{0} & =\sum_{k, \sigma} \epsilon_{k} a_{k, \sigma}^{\dagger} a_{k, \sigma}+\sum_{k, \sigma} \epsilon_{k} b_{k, \sigma}^{\dagger} b_{k, \sigma} \\
& +\sum_{\ell}^{N_{y}} \sum_{k, \sigma} V_{k, \ell, j}^{L} d_{\ell, j, \sigma}^{\dagger} a_{k, \sigma}+\sum_{\ell}^{N_{y}} \sum_{k, \sigma} V_{k, \ell, j}^{R} d_{\ell, j, \sigma}^{\dagger} b_{k, \sigma}+H . c .
\end{aligned}
$$

The first two terms of Eq. (1) describe the free electron gas in the left and right electrodes. $a_{k, \sigma}^{\dagger}\left(b_{k, \sigma}^{\dagger}\right)$ creates 
an electron of momentum $k$ and spin $\sigma$ with energy $\epsilon_{k}$ in the left (right) electrode. $V_{k, \ell, j}^{L}\left(V_{k, \ell, j}^{R}\right)$ describes the coupling between the left (right) lead with its adjacent QD in the $\ell$ th row, which counts from 1 to $N_{y}$.

$$
\begin{aligned}
& H_{Q D}=\sum_{\ell, j, \sigma} E_{\ell, j} d_{\ell, j, \sigma}^{\dagger} d_{\ell, j, \sigma} \\
& +\sum_{\sigma} \sum_{\ell 1, \ell 2}^{N_{y}} \sum_{j 1, j 2}^{N_{x}} t_{\ell 1, \ell 2, j 1, j 2} d_{\ell 1, j 1, \sigma}^{\dagger} d_{\ell 2, j 2, \sigma}+H . c, \\
& t_{\ell 1, \ell 2, j 1, j 2}=\left\{\begin{array}{l}
-t_{y} \text { if } j 1=j 2,|\ell 1-\ell 2|=1 \\
-t_{x} \text { if } \ell 1=\ell 2,|j 1-j 2|=1
\end{array},\right.
\end{aligned}
$$

where $E_{\ell, j}$ is the energy level of QD in the $\ell$-th row and $j$-th column. The spin-independent $t_{\ell 1, \ell 2, j 1, j 2}$ describes the electron hopping strength, which is limited to the nearest neighboring sites. $d_{\ell 1, j 1, \sigma}^{\dagger}\left(d_{\ell 2, j 2, \sigma}\right)$ creates (destroys) one electron in the QD at the $\ell$ th row and $j$ th column. If the wave functions of the electrons in each QD are localized, the electron Coulomb interactions are strong. Their effects on electron transport are significant in the scenario of weak hopping strengths. 22] On the other hand, the wave functions of the electrons are delocalized in the scenario of strong hopping strengths to form minibands; hence their weak electron Coulomb interactions can be ignored.

To study the transport properties of a 2D QD array junction connected to electrodes, it is convenient to use the Keldysh-Green's function technique 21,23]. Electron and heat currents leaving electrodes can be expressed as

$$
J=\frac{2 e}{h} \int d \varepsilon T_{L R}(\varepsilon)\left[f_{L}(\varepsilon)-f_{R}(\varepsilon)\right]
$$

and

$$
\begin{aligned}
& Q_{e, L(R)} \\
= & \frac{ \pm 2}{h} \int d \varepsilon T_{L R}(\varepsilon)\left(\varepsilon-\mu_{L(R)}\right)\left[f_{L}(\varepsilon)-f_{R}(\varepsilon)\right]
\end{aligned}
$$

where $f_{\alpha}(\varepsilon)=1 /\left\{\exp \left[\left(\varepsilon-\mu_{\alpha}\right) / k_{B} T_{\alpha}\right]+1\right\}$ denotes the Fermi distribution function for the $\alpha$-th electrode, where $\mu_{\alpha}$ and $T_{\alpha}$ are the chemical potential and the temperature of the $\alpha$ electrode. $e, h$, and $k_{B}$ denote the electron charge, the Planck's constant, and the Boltzmann constant, in that order. $T_{L R}(\varepsilon)$ denotes the transmission coefficient of a 2D QD array connected to electrodes, which can be solved by the formula $T_{L R}(\varepsilon)=$ $4 \operatorname{Tr}\left[\hat{\Gamma}_{L} \hat{G}_{D, A}^{r}(\varepsilon) \hat{\Gamma}_{R} \hat{G}_{D, A}^{a}(\varepsilon)\right]$, where the matrix of tunneling rates $\left(\hat{\Gamma}_{L}\right.$ and $\left.\hat{\Gamma}_{R}\right)$ and Green's functions $\left(\hat{G}_{D, A}^{r}(\varepsilon)\right.$ and $\left.\hat{G}_{D, A}^{a}(\varepsilon)\right)$ can be constructed by coding. 24]

The electrical conductance $\left(G_{e}\right)$, Seebeck coefficient $(S)$ and electron thermal conductance $\left(\kappa_{e}\right)$ can be evaluated by using Eqs. (4) and (5) with a small applied bias $\Delta V=\left(\mu_{L}-\mu_{R}\right) / e$ and cross-junction temperature difference $\Delta T=T_{L}-T_{R}$. We obtain these thermoelectric coefficients $G_{e}=e^{2} \mathcal{L}_{0}, S=-\mathcal{L}_{1} /\left(e T \mathcal{L}_{0}\right)$ and
$\kappa_{e}=\frac{1}{T}\left(\mathcal{L}_{2}-\mathcal{L}_{1}^{2} / \mathcal{L}_{0}\right) . \mathcal{L}_{n}$ is given by

$$
\mathcal{L}_{n}=\frac{2}{h} \int d \varepsilon T_{L R}(\varepsilon)\left(\varepsilon-E_{F}\right)^{n} \frac{\partial f(\varepsilon)}{\partial E_{F}},
$$

where $f(\varepsilon)=1 /\left(\exp ^{\left(\varepsilon-E_{F}\right) / k_{B} T}+1\right)$ is the Fermi distribution function of electrodes at equilibrium temperature T.

\section{RESULTS AND DISCUSSION}

According to Eq. (6), transmission coefficient plays a significant role for electron transport between the electrodes. To illustrate the electronic states of finite 2D QD array, we have calculated and shown in Fig. 2 transmission coefficient $T_{L R}(\varepsilon)$ as a function of $\varepsilon$ for different tunneling rates $\left(\Gamma_{L(R), \ell, j}(\varepsilon)=2 \pi \sum_{k}\left|V_{k, \ell, j}^{L(R)}\right|^{2} \delta\left(\varepsilon-\varepsilon_{k}\right)=\right.$ $\left.\Gamma_{t}\right)$. A square lattice with homogenous electron hopping strengths $t_{x}=t_{y}=t_{c}=6 \Gamma_{0}$ and site-independent QD energy level $E_{\ell, j}=E_{0}=E_{F}$ has been considered in the calculation of $T_{L R}(\varepsilon)$. All physical parameters are in units of $\Gamma_{0}$. In Fig. 2(a), $T_{L R}(\varepsilon)$ reveals the tunneling probability of the electrons of the electrodes through the electronic states of 2D QD array, those energy is described by $\varepsilon=E_{0}-2 t_{c}\left(\cos \left(\frac{n_{x} \pi}{N_{x}+1}\right)+\cos \left(\frac{n_{y} \pi}{N_{y}+1}\right)\right)$, where $n_{x}=1,2, . . N_{x}$ and $n_{y}=1,2, . . N_{y}$. Because the QD array is connected to the electrodes, these electronic states have inhomogeneous broadening. They are also restricted within the range between $-4 t_{c}$ and $4 t_{c}$. We can tune the distribution of electronic states by changing $N, t_{c}$ and $\Gamma_{t}$.
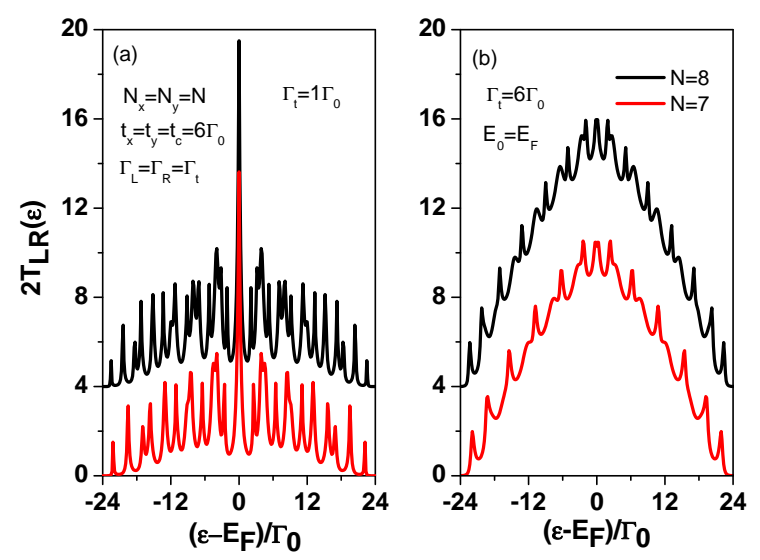

FIG. 2: Transmission coefficient $T_{L R}(\varepsilon)$ as a function of $\varepsilon$ for different $N$ values at $t_{x}=t_{y}=t_{c}=6 \Gamma_{0}$ and $E_{0}=E_{F}$. Diagrams (a) and (b) consider tunneling rates $\Gamma_{L}=\Gamma_{R}=$ $\Gamma_{t}=1 \Gamma_{0}$ and $\Gamma_{t}=6 \Gamma_{0}$, respectively. To prevent the curves from overlapping each other, we shifted the curve of $\mathrm{N}=8$.

From Eqs. (4) and (5), the maximum electron current and heat current occur at $T_{L R}(\varepsilon)$ with the maximum area. The authors of Ref. [24] proved two results: the maximum area of $T_{L R}(\varepsilon)$ can be reached at 
the condition of $\Gamma_{t}=t_{c}$ and the maximum area increases with increasing $N$, as seen in Fig. 2(b). Note that the 2D tight-binding electronic states show the Van Hove singularity in the density of states (DOS) as $N \rightarrow \infty$ (DOS diverges at $E_{0}$ ). At zero temperature, the electrical conductance is given by the transmission coefficient $G_{e}=\frac{2 e^{2}}{h} T_{L R}\left(E_{F}\right)$. We now clarify how the electronic states influence the thermoelectric coefficients of a finite 2D QD array.

Fig. 3 shows the calculated $G_{e}, \kappa_{e}$ and Lorenz number $\left(L_{0}=\kappa_{e} /\left(T G_{e}\right)\right.$ at functions of the QD energy level for various values of $\Gamma_{t}$ at $k_{B} T=1 \Gamma_{0}, t_{c}=6 \Gamma_{0}$ and $N=8$. As a result of temperature effect $\left(\frac{\partial f(\varepsilon)}{\partial E_{F}}\right)$, the electronic states shown in Fig. 2(a) can not be resolved in Fig. $3(\mathrm{a})$. It is not easy to justify a finite $2 \mathrm{D} \mathrm{QD}$ array in the band-like or molecule-like situation from $G_{e}$ at finite temperature, especially at high temperatures. The curves of $\kappa_{e}$ in Fig. 3(b) are similar to those of $G_{e}$. According to the Wiedemann-Franz law, $L_{0} /\left(k_{B}^{2} / e^{2}\right)=\frac{\pi^{2}}{3}$ is a temperature-independent quantity. In Fig. $3(\mathrm{c})$, the $L_{0}$ curve corresponding to $\Gamma_{t}=6 \Gamma_{0}$ is approximately $\pi^{2} / 3$. For comparison, we also add the calculated $G_{e}, \kappa_{e}$ and $L_{0}$ for 1D QD array with $N_{x}=100$ and $t_{c}=\Gamma_{t}=12 \Gamma_{0}$ (the band width of $48 \Gamma_{0}$ in this $1 \mathrm{D}$ miniband). As seen in Fig. $3(\mathrm{c}), 1 \mathrm{D}$ QD array yields a Lorenz number $L_{0}=\frac{k_{B}^{2}}{e^{2}} \frac{\pi^{2}}{3}$ between $-10 \Gamma_{0} \leq \Delta \leq 10 \Gamma_{0}$. This can be regarded as a manifested band-like transport.

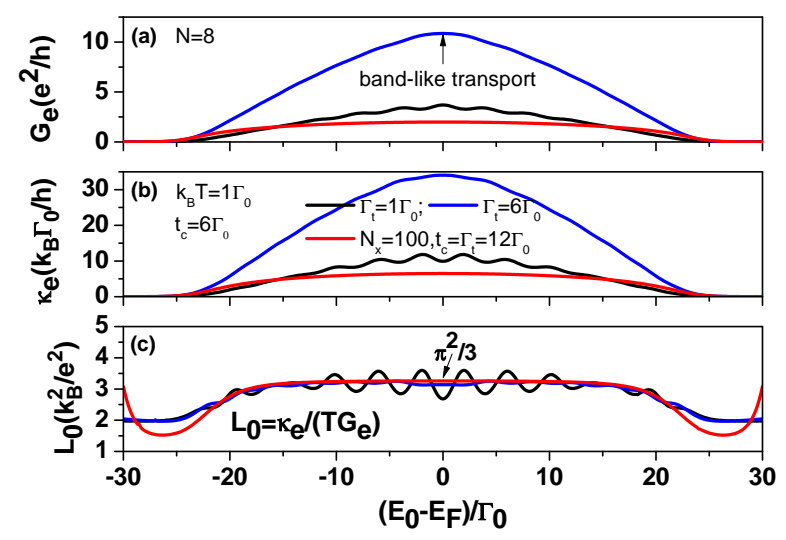

FIG. 3: (a) Electrical conductance $G_{e}$, (b) electron heat conductance $\kappa_{e}$ and (c) Lorenz number $\left(L_{0}=\kappa_{e} /\left(T G_{e}\right)\right)$ as functions of $\Delta=E_{0}-E_{F}$ for various $\Gamma_{t}$ values at $k_{B} T=1 \Gamma_{0}$, $t_{c}=6 \Gamma_{0}$ and $N=8$. The red curves correspond to a $1 \mathrm{D} \mathrm{QD}$ array with $N_{x}=100, t_{c}=\Gamma_{t}=12 \Gamma_{0}$ and $k_{B} T=1 \Gamma_{0}$.

Furthermore, we have calculated $G_{e}, \kappa_{e}$ and $L_{0}$ for $2 \mathrm{D}$ QD array with $N=8$ as functions of temperature for different $\Gamma_{t}$ values at $E_{0}=E_{F}$ and $t_{c}=6 \Gamma_{0}$ in Fig. 4 . The red curves are the results of the 1D QD array corresponding to those of Fig. 3. One-dimensional QD arrays have a temperature-independent $G_{e}$ and a linear temperaturedependent $\kappa_{e}$. As a consequence, $L_{0}=\kappa_{e} /\left(T G_{e}\right)$ leads to a temperature-independent behavior. According to the results of Figs. 3 and 4, the thermoelectric properties of $2 \mathrm{D}$ QD arrays with $t_{c}=6 \Gamma_{0}, \Gamma_{t}=6 \Gamma_{0}$ and $N=8$ are very similar to those of $1 \mathrm{D} \mathrm{QD}$ arrays with minibands. We deduce that finite 2D QD arrays have band-like characteristics when $t_{c}=\Gamma_{t}=6 \Gamma_{0}$ and $N=8$.

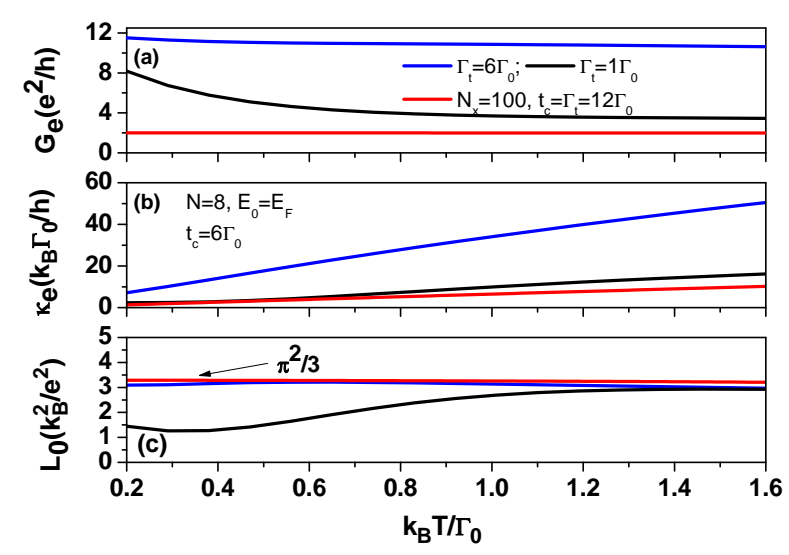

FIG. 4: (a) Electrical conductance, (b) electron heat conductance and (c) Lorenz number as functions of temperature for various $\Gamma_{t}$ values at $E_{0}=E_{F}, t_{c}=6 \Gamma_{0}$ and $N=8$. The red curves correspond to those of Fig. 3 with $E_{0}=E_{F}$.

Because many thermoelectric devices operate at high temperatures, it is important to examine the power factor of 2D QD arrays in this regime. In Figs. 2-4 we have focused on the electron transport in resonant tunneling procedure (RTP) in which the Seebeck coefficient is very small. To obtain a large $P F$ value at high temperature, we considered the electron transport in the thermionic-assisted tunneling procedure (TATP) where the band center $\left(E_{0}\right)$ is far away from the Fermi level $E_{F}$ in the electrodes. In Fig. 5, we have calculated $G_{e}, S, P F$ and $L_{0}$ as functions of temperature for various $t_{c}$ values at $E_{0}-E_{F}=30 \Gamma_{0}$. Because the maximum $T_{L R}(\varepsilon)$ area occurs at $\Gamma_{t}=t_{c}$, we adopted this condition for all the subsequent steps. As seen in Fig. 5(a), $G_{e}$ is vanishingly small at low temperature due to the electronic states of 2D QD arrays being kept a certain distance from the $E_{F}$. The enhancement of $G_{e}$ with increasing temperature is a typical characteristic arising from the TATP. To understand the temperature behavior of $G_{e}$ at $\Gamma_{t}=t_{c}=1 \Gamma_{0}$, we have the expression of $G_{e, \text { atom }}=\frac{e^{2}}{h} \frac{\pi \Gamma_{t}}{2 k_{B} T \cosh ^{2}\left(\left(E_{0}-E_{F}\right) /\left(2 k_{B} T\right)\right)}$ when the transmission coefficient is approximated as $T_{L R}(\varepsilon)=4 \Gamma_{t}^{2} /\left(\left(\varepsilon-E_{0}\right)^{2}+\left(2 \Gamma_{t}\right)^{2}\right)$ in Eq. (6). In addition, $S_{\text {atom }}=-\Delta / T=-\left(E_{0}-E_{F}\right) / T$, which explains the behavior of $S$ at $t_{c}=1 \Gamma_{0}$ and $k_{B} T \geq 2 \Gamma_{0}$ in Fig. $5(\mathrm{~b})$. Although $G_{e}$ is highly enhanced with increasing $t_{c}$, $S$ is not so sensitive to $t_{c}$ for $k_{B} T \geq 10 \Gamma_{0}$. This explains why the trend of maximum $P F$ for $t_{c}$ shown in Fig. 5(c) is determined by $G_{e}$. In Fig. $5(\mathrm{~d})$, three $L_{0}$ curves violate the Wiedemann-Franz law. Note that $\Gamma_{t}=t_{c}=6 \Gamma_{0}$ provides the band-like characteristic (see Fig. 3(c)). 

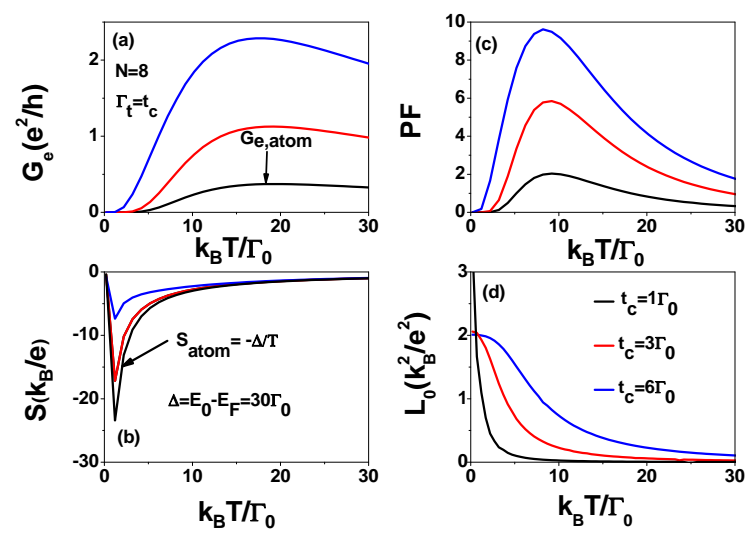

FIG. 5: (a) Electrical conductance, (b) Seeback coefficient, (c) power factor $\left(P F=S^{2} G_{e}\right)$ and (d) Lorentz number as functions of $T$ for various $t_{c}$ values at $E_{0}-E_{F}=30 \Gamma_{0}$ and $N=8$. Meanwhile, we have adopted $\Gamma_{t}=t_{c}$.

Fig. 6 shows the calculated $G_{e}, S$ and $P F$ as functions of $E_{0}$ for various $t_{c}$ values at $k_{B} T=25 \Gamma_{0}$ to reveal the effect of the band center. As seen in Fig. 6(a), $G_{e}$ has a maximum value when the band center $\left(E_{0}\right)$ is located at $E_{F}$. The Seeback coefficients in Fig. 6(b) are zero at $E_{0}=E_{F}$. It is attributed to the symmetrical distribution of the electrons and holes on the electronic states of the 2D QD array. Here, the holes are defined as the empty states below $E_{F}$. For $k_{B} T=25 \Gamma_{0}$, the Seebeck coefficients are well described by $S_{\text {atom }}=-\Delta / T$. We find that the maximum values of $P F$ occur near $\Delta=60 \Gamma_{0}$, as indicated in Fig. 6(c). When approaching the atomic limit $\left(t_{c} \rightarrow 0\right)$, one can prove that the optimization of $P F$ is given by $\Delta / k_{B} T=2.4$. The results of Fig. 6(c) imply that 2D QD arrays with minibands $\left(t_{c}=6 \Gamma_{0}\right)$ preserve the atomic thermoelectric properties when the band center is far away from the $E_{F}$ of the electrodes. In Fig. 2(b), $T_{L R}(\varepsilon)$ depends on $N$. Therefore, we add in Fig. 6 the curves with triangle marks for $N=7$ and $t_{c}=6 \Gamma_{0}$. From the curves of $N=7$ and $N=8$, we see that the enhancement of $G_{e}$ resulting from the increase of electronic states does not suppress $S$. It is worthy noting that a single 1D QD array does not exist such a behavior. We reinvestigate $P F$ as functions of $t_{c}$ for $N=7,8$ at $\Delta=60 \Gamma_{0}$ and $k_{B} T=25 \Gamma_{0}$ in Fig. 6(d). $P F$ is a linear function of $t_{c}$ as $t_{c} \leq 6 \Gamma_{0}$. Meanwhile, the maximum $P F$ is given by $t_{c}=\Delta / 4$. The red curves represent the case where $t_{y}=0$ to clarify the geometer effects. When $t_{c} \leq 6 \Gamma_{0}$, the geometry effects can be ignored.

Because $T_{L R}(\varepsilon)$ lacks an analytical form, it is not easy to illustrate the complex behavior of $P F$ shown in Fig. $6(d)$. If we make the assumption that minibands have homogenous electronic states and consider the squareform $T_{L R}(\varepsilon)$ given by

$$
T_{L R}(\varepsilon)= \begin{cases}N_{y} & \text { if }-2 t_{c} \leq \varepsilon-E_{0} \leq 2 t_{c} \\ 0 & \text { otherwise }\end{cases}
$$

the analytical forms of $G_{e}$ and $S$ can be derived as

$$
G_{e}=\frac{2 e^{2} N_{y}}{h}\left(\tanh \left(y_{1}\right)-\tanh \left(y_{2}\right)\right)
$$

and

$$
S=\frac{2 e k_{B} N_{y}}{h} \frac{\left(S_{1}\left(y_{1}\right)-S_{2}\left(y_{2}\right)\right)}{G_{e}}
$$

where $S_{i}\left(y_{i}\right)=y_{i} \tanh \left(y_{i}\right)-\log \left(\cosh \left(y_{i}\right)\right), y_{1}=\frac{\Delta+2 t_{c}}{2 k_{B} T}$ and $y_{2}=\frac{\Delta-2 t_{c}}{2 k_{B} T}$. In Fig $6(\mathrm{~d})$, the blue curves are calculated by using Eqs. (8) and (9). Because Eq. (7) considers $N_{y}$ 1D QD arrays with homogenous electric states, it is expected that the $P F$ given by Eqs. (8) and (9) is overestimated. However, Eqs. (8) and (9) provide a clear picture that the enhancement of $P F$ follows the enhancement of $G_{e}$. Meanwhile, $S \approx S_{\text {atom }}$ as long as $\frac{t_{c}}{k_{B} T}<0.25$ and $\frac{\Delta}{k_{B} T} \geq 2.4$. We deduce that $G_{e}$ in a band-like transport situation and $S$ in an atomic-like situation can coexist for finite 2D QD arrays with $t_{c}=6 \Gamma_{0}$ and $\Delta=60 \Gamma_{0}$ at $k_{B} T=25 \Gamma_{0}$. If we set $\Gamma_{0}=1 \mathrm{meV}$, then our analysis in Fig. 6 becomes a very useful guideline for thermoelectric devices operated at room temperature.
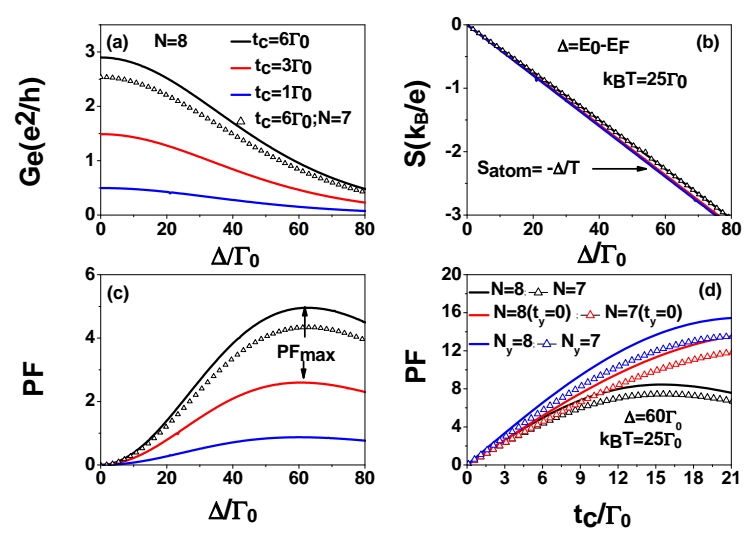

FIG. 6: (a) Electrical conductance, (b) Seebeck coefficient and (c) power factor as functions of $E_{0}$ for different $t_{c}$ values at $N=8$ and $k_{B} T=25 \Gamma_{0}$. The curves with black triangle marks correspond to the case of $N=7$ and $t_{c}=6 \Gamma_{0}$.(d) $P F$ as functions of $t_{c}$ for $\Delta=60 \Gamma_{0}$ and $k_{B} T=25 \Gamma_{0}$. The red curves correspond to $t_{y}=0$. The blue curves are calculated using Eq. (7).

\section{CONCLUSION}

We have theoretically investigated the thermoelectric properties of 2D QD arrays. In RTP, the Lorenz number with a value near $\pi^{2} / 3$ and a temperature-independent behavior demonstrates that 2D QD arrays with $t_{c}=\Gamma_{t}=$ $6 \Gamma_{0}$ and $N=8$ indeed form minibands. When this miniband center is far away from the Fermi level of the electrodes, TATP dominates the electron transport between 
the electrodes and $L_{0}$ violates the Wiedemann-Franz law. In TATP, $G_{e}$ is enhanced as the number of electronic states increases, whereas the $S$ values remain in an atomlike situation. This is a remarkable property that would lead to a high-efficiency thermoelectric devices made of QDs with large electrical power output. This interesting phenomenon exists not only for 2D QD arrays with square-lattices but also triangular-lattices, which will be reported in elsewhere.

Acknowledgments

This work was supported under Contract No. MOST
107-2112-M-008 -023MY2

E-mail address: mtkuo@ee.ncu.edu.tw

\section{Data Availability Statements}

The data that supports the findings of this study are available within the article.
1 L. J. Guo, E. Leobandung, and S. Y. Chou, Science 275, 649 (1997).

2 H. W. C Postma, T. Teepen, Z. Yao, M. Grifoni, and C. Dekker, Science 293, 76 (2001).

3 S. Kubatkin, A. Danilov, M. Hjort, J. Cornil, J. L. Bredas, N. Stuhr-Hansen, P. Hedegard, and T. Bjornholm, Nature 425, 698 (2003).

4 P. Michler, A. Imamoglu, M. D. Mason, P. J. Carson, G. F. Strouse, and S. K. Buratto, Nature 406, 968 (2000).

${ }^{5}$ C. Santori, D. Fattal, J. Vuckovic, G. S. Solomon, and Y. Yamamoto, Nature 419, 549 (2002).

${ }^{6}$ W. H. Chang, W. Y. Chen, H. S. Chang, T. P. Hsieh, J. I. Chyi, and T. M. Hsu, Phys. Rev. Lett. 96, 117401 (2006).

7 S. Gustavsson, M. Studer, R. Leturcq, T. Ihn, K. Ensslin, D. C. Driscoll and A. C. Gossard, Phys. Rev. Lett. 99, 206804 (2007).

8 M. Josefsson, A. Svilans, A. M. Burke, E. A. Hoffmann, S. Fahlvik,C. Thelander,M. Leijnse, H. Linke, Nature Nanotechnology 13, 920 (2018).

9 C. R. Kagan and C. B. Murry, Nature Nanotechnology 10, 1013 (2015).

10 T. C. Harman, P. J. Taylor, M. P. Walsh, and B. E. LaForge, Science 297, 2229 (2002).

11 E. Talgorn, Y. Gao, M. Aerts1, L. T. Kunneman, J. M. Schins, T. J. Savenije, Marijn A. van Huis, Herre S. J. van der Zant, Arjan J. Houtepen and Laurens D. A. Siebbeles, Nature Nanotechnology 6,733 (2011).
12 David. M.-T. Kuo and Y. C. Chang, Phys. Rev. B 81, 205321 (2010).

13 R. S. Whitney, Phys. Rev. Lett. 112, 130601 (2014).

14 David M. T. Kuo and Y. C. Chang, Physica E 115, 113671 (2020).

15 G. Chen, Phys. Rev. B 57, 14958 (1998).

16 G. Chen, M. S. Dresselhaus, G. Dresselhaus, J. P. Fleurial, and T. Caillat, International Materials Reviews, 48, 45 (2003).

17 G. D. Mahan, L. M. Woods, Phys. Rev. Lett. 80, 4016 (1998).

18 L. D. Lhao, S. H. Lo, Y. Zhang, H. Sun, G. Tan, C. Uher, C. Wolverton, V. P. Dravid and M. G. Kanatzidis, Nature 508, 373 (2014).

19 C. Chang, M. Mu, D. He, Y. Pei, and C. F. Wu et al., Science 360, 778 (2018).

20 D. D. Fan, H. J. Liu, L. Cheng, P. H. Jiang, J. Shi and X. F. Tang, Appl. Phys. Lett. 105, 133113 (2014).

21 H. Haug and A. P. Jauho, Quantum Kinetics in Transport and Optics of Semiconductors (Springer, Heidelberg, 1996).

22 D. M. T. Kuo, C. C. Chen and Y. C. Chang, Phys. Rev. B 95, 075432 (2017).

23 Y. Meir and N. S. Wingreen, Phys. Rev. Lett. 68, 2512 (1992).

24 D. M. T. Kuo, AIP Advances 10, 045222 (2020). 